\title{
Ciclo de Mejora en el Aula en la Asignatura "Afecciones Médicas I. 2o curso. Grado de Fisioterapia y Doble Grado en Fisioterapia y Ciencias del Deporte"
}

\section{The improvement cicle in Classroom in the Subject “Medical Disorders I". 2nd grade. Degree in Physiotherapy and Double Degree in Physiotherapy and Sports Sciences"}

Ma Angeles VÁZqueZ GÁmeZ

ORCID: https://orcid.org/0000-0001-7689-6999 Universidad de Sevilla,

Facultad de Medicina.

Departamento de Medicina

Correo electrónico: mavazquez@us.es

Fecha de recepción: 21 de noviembre de 2019

Fecha de aceptación: 22 de noviembre de 2019

DOI: http://dx.doi.org/10.12795/9788447221912.072

Pp.: 1658-1677 


\section{Resumen}

Este Ciclo de Mejora en el Aula (CIMA) se desarrolla sobre una asignatura TRONCAL y cuatrimestral, del segundo curso del Grado en Fisioterapia y del Doble Grado de en Fisioterapia y Ciencias del Deporte. Su contenido se fundamenta en el conocimiento de las patologías más frecuentes que llegan al fisioterapeuta, sus causas, mecanismos fisiopatológicos, cómo se manifiestan clínicamente y como se tratan. Para ello, hemos considerado importante que el alumno se aproxime lo más posible a la realidad, trabajando con casos clínicos reales o ficticios, que permitan englobar los conocimientos necesarios para abordarlos de manera eficiente, incluyendo las competencias transversales que se describen para esta asignatura. Para evaluar el proceso utilizamos un cuestionario inicial con los puntos básicos de donde queremos que parta nuestro aprendizaje y un cuestionario final para conocer los logros obtenidos, empleando escaleras de aprendizaje para la evaluación del proceso. Durante el desarrollo de este CIMA, se diseñarán dinámicas diferentes para conseguir los objetivos propuestos y la autonomía del estudiante.

Palabras claves: Afecciones Médicas I, Grado en Fisioterapia, Docencia Universitaria, Experimentación Docente Universitaria.

\section{Abstract}

This Cycle of Improvement in the Classroom (CIMA) is developed on a subject TRONCAL and four-monthly, of the second course of the Degree in Physiotherapy and the Double Degree in Physiotherapy and Sports Sciences. Its content is based on the knowledge of the most frequent pathologies treated by the physiotherapist, their causes, physiopathological mechanisms, how they are clinically manifested and how they are treated. To this end, we have considered it important for the student to approach reality as closely as possible, working with real or fictitious clinical cases that allow the necessary knowledge to be included in order to be tackled efficiently, including the transversal competences that are described for this subject. To evaluate the process we use an initial questionnaire with the basic points from which we want our learning to start and a final questionnaire to know the achievements obtained, using learning ladders for the evaluation of the process. During the development of this CIMA, different dynamics will be designed to achieve the proposed objectives and the autonomy of the student.

Key words: “Medical Disorders I", “Physiotherapy Degree”, “University Teaching","University Teaching Experimentation" 


\section{Breve descripción del contexto de la asignatura:}

Este nuevo CIMA se implanta en el Grado de Fisioterapia y en el Doble Grado de Fisioterapia y Ciencias del Deporte. Concretamente en la asignatura Afecciones Médicas I, la misma en la que se realizó el anterior CIMA. Se imparte en 2 o curso de este Grado, concretamente en el primer cuatrimestre. Es una asignatura troncal, que constituye la base sobre la que se van a desarrollar las distintas técnicas y procedimientos de fisioterapia, que constituyen el grueso de este Grado. La asignatura Afecciones Médicas I, consta de una parte teórica, una parte práctica y seminarios en aula. Este CIMA está incluido en el contenido teórico de la asignatura. La parte teórica consta de 52 temas, agrupados en 6 bloques temáticos. Este contenido teórico se imparte entre 3 profesoras, que compartimos la materia. La asignatura se imparte 4 días a la semana hasta la semana 20 y a partir de la semana 21, 3 horas semanales (1 hora/día de lunes a jueves). El número de alumnos es de 75 por aula (tenemos 2 grupos) y la media de alumnos que asisten a clase es de unos 30-35 en cada grupo. Tanto el CIMA previo, como el que se va a proponer, se llevarán a cabo concretamente en el IV bloque temático: Fisiopatología y Enfermedades del Aparato Circulatorio), que incluye 13 temas del programa.

El curso pasado, ya realicé un CIMA previo en esta asignatura (presentados en la convocatoria anterior de la REFID). En esta nueva convocatoria, tengo intención de mejorar las actividades realizadas el curso pasado, y aumentar progresivamente el número de temas incluidos en la innovación. El CIMA que se propone, perteneciente a la tipología "encadenado", tiene como objetivo completar el CIMA previo que englobaba 4 temas de este apartado (6h docentes en total).

Jornadas de Formación e Innovación Docente del Profesorado | № 2 (2019) Esta obra se distribuye con la licencia Creative Commons Reconocimiento-NoComercial-SinObraDerivada Internacional (CC BY-NC-ND 4.0.) 


\section{Conexión con el proceso previo}

Dado que la experiencia del CIMA previo fue muy positiva en general, (me permitió entender la importancia de conocer los modelos mentales de los alumnos y el papel de la empatía entre profesor y alumno para la consecución de objetivos docentes; comprobé la trascendencia de planificar la docencia y las ventajas de priorizar los procedimientos frente a la teoría; Aprendí el valor del control del tiempo de trabajo y la construcción de unas preguntas clave adecuadas; y pude comprobar la relevancia de escoger un método de evaluación adecuado). Por ello, y teniendo en cuenta todas estas consideraciones, me propongo ampliar este CIMA con la inclusión de 3 horas más de docencia (2 temas), incluidas también en el IV bloque temático de la asignatura Afecciones Médicas I, que integren y conecten con las patologías vistas en el CIMA previo y que aproximen al alumno a la realidad clínica.

La dinámica de trabajo, aunque en algunos apartados mantendrá la similitud con el CIMA anterior, presenta algunas variaciones en la metodología empleada para trabajar algunos aspectos del tema, que se irán comentando. La ampliación de este CIMA, conecta con temas que tiene una importante relación con los temas vistos en el CIMA anterior, tales como son el sistema venoso y el sistema linfático.

El objetivo principal de estos nuevos temas dentro del bloque de Fisiopatología y Enfermedades del Aparato Circulatorio, es que el alumno aprenda en qué circunstancias no se puede llevar a cabo la terapia manipulativa en extremidades inferiores que presentan diferentes patologías, ya que puede tener consecuencias graves para el paciente, así como saber diferenciar las características de los diferentes tipos de edemas más comunes en la población.

Siguiendo la mecánica previamente utilizada, partiremos de preguntas claves que generen curiosidad al alumno y les permita conectar con el resto del contenido. 
En las figura 1 se describe el mapa de contenido

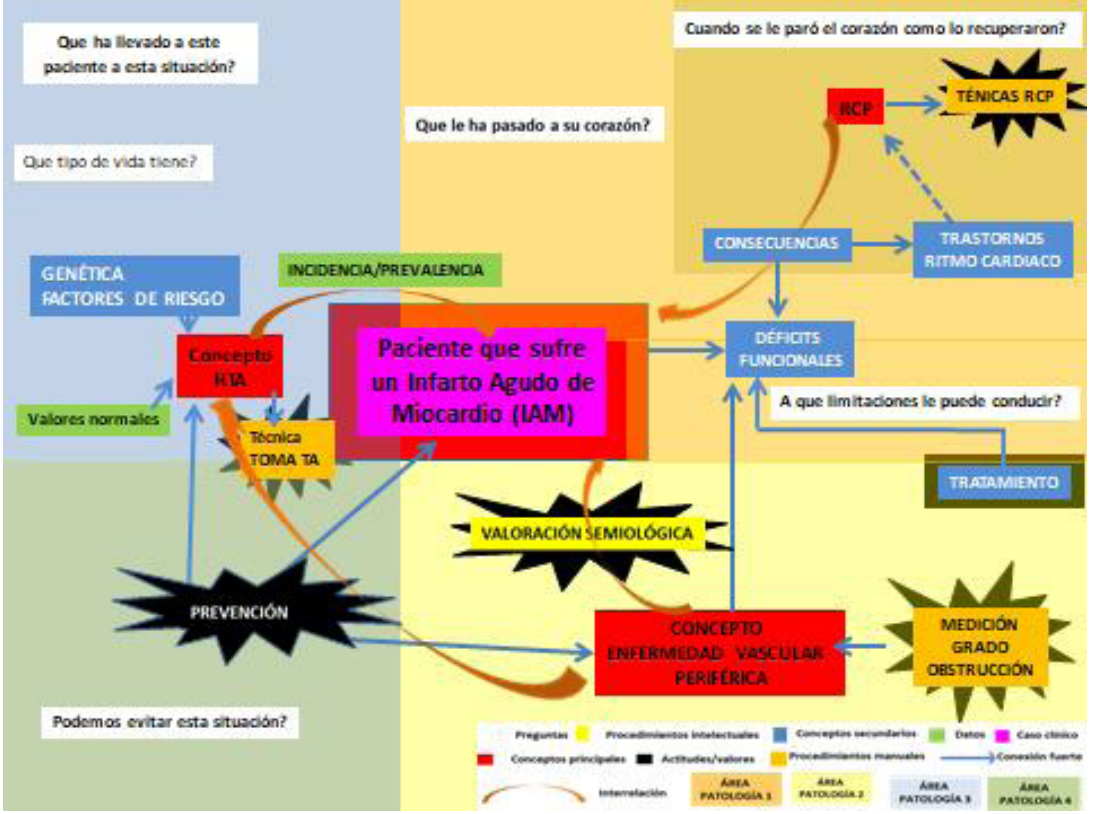

Fig. 1: Mapa de contenidos del CIMA previo.

La Fig. 2 representa el mapa propuesto para este CIMA, con la nueva ampliación.

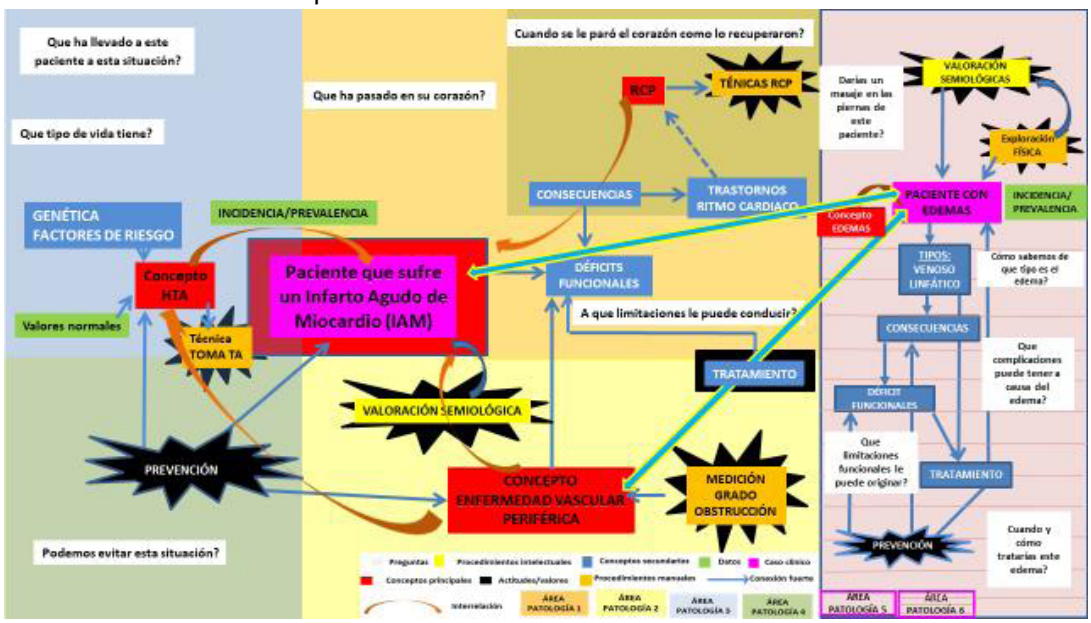

Fig. 2 La zona con fondo rosa, muestra el nuevo CIMA y su relación con el CIMA previo.

Jornadas de Formación e Innovación Docente del Profesorado | № 2 (2019) (C) Esta obra se distribuye con la licencia Creative Commons 
Como se puede observar, el nuevo CIMA propuesto, sigue el mismo patrón que el que se llevó a cabo el año anterior. Partiendo de un caso clínico que se proyecta en un video (de un paciente con un IAM), vamos conectando con las diferentes patologías que se incluyen a este bloque temático de patología cardiovascular y que tienen un posible nexo de unión con el caso.

En el curso anterior, el CIMA propuesto conectaba mediante diferentes preguntas con los contenidos que consideramos esenciales para el conocimiento del alumno dentro de este bloque. Así, planteándonos el motivo que ha llevado al paciente a esta situación, nos permite entrar en los factores de riesgo para provocar un IAM y dentro de ellos, como factor con mayor peso, tocar la HTA (Temas 2223 del programa). Con la pregunta que ha pasado en su corazón, nos permite evaluar los mecanismos fisiopatológicos que llevan a la cardiopatía isquémica (IAM) y conocer cómo se manifiestan clínicamente estas alteraciones (valoración semiológica). (Temas 17 y 19).

Nuestro caso clínico sufre una parada cardiaca como complicación de su infarto, lo que nos lleva a sumergirnos en el tema de la resucitación cardiopulmonar (RCP) (Tema 28 del programa)

El hecho de que nuestro paciente sufra una enfermedad vascular arterial periférica, es una causa de IAM. Así conectamos las 2 enfermedades.

La pregunta de qué limitaciones sufren estos pacientes, permitiría conectar con el tratamiento de las discapacidades que pueden presentarse tras sufrir un IAM y que serían objeto de asignaturas de fisioterapia, lo cual completaría el ciclo de mejora docente, favoreciendo la integración de asignaturas. Las distintas áreas temáticas están plasmadas sobre fondos de colores diferentes.

De esta manera, y partiendo de un caso clínico, hemos aunado 4 patologías que se veían antes de forma 
independiente e inconexa, integrando de esta manera la información.

En este nuevo CIMA, ampliamos otros 2 temas (3 horas) de este bloque temático: la patología vascular periférica venosa y la patología linfática. De nuevo, y partiendo del caso clínico inicial, en la que el paciente presenta edemas tras sufrir el IAM, hacemos el diagnóstico diferencial entre los diferentes tipos de edemas, para reconocerlos, diferenciarlos y saber cuándo se pueden tratar y cuando no. Esto nos permite conocer cómo se clasifican, que consecuencias tienen, que limitaciones pueden originar y como prevenirlos.

\section{Modelo Metodológico Ideal y Posible:}

El modelo metodológico ideal sería el que mejor simulase la realidad profesional que van a tener los estudiantes. Por tanto, eminentemente práctico. Lo ideal sería que el alumno pudiese acceder a pacientes reales, interrelacionar con ellos y trabajar, supervisados por los profesores que imparten asignaturas afines a Afecciones Médicas I, sobre las patologías que presenten.

En las figuras 3 y 4 se describen los modelos metodológicos: ideal y posible

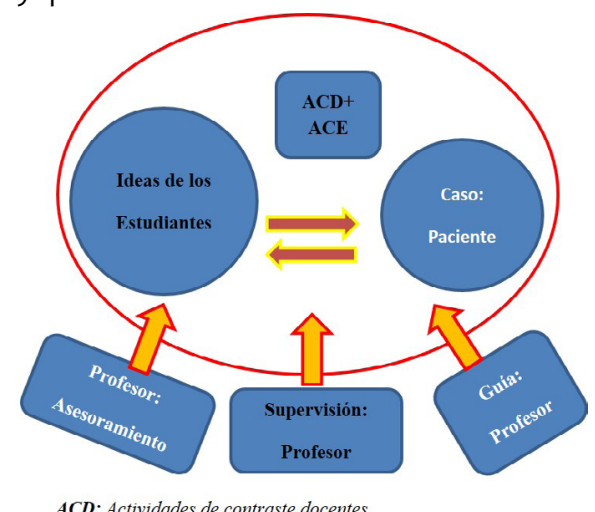

ACE: Actividades de contraste basadas en experimentos

Fig. 3 Modelo metodológico ideal

Jornadas de Formación e Innovación Docente del Profesorado | № 2 (2019) Esta obra se distribuye con la licencia Creative Commons 
Hasta el momento actual, el modelo metodológico ideal es imposible llevarlo a cabo. En este curso (2o curso), los alumnos aún no están en contacto con pacientes, es dificil coordinar horarios y profesorado y estructurar las asignaturas afines para trabajar en colaboración. Por tanto, hay que buscar alternativas para llevarles al aula.

Por ello, en el modelo metodológico posible que nos proponemos, hemos optado por dinamizar la clase con diferentes actividades que lleven a converger todos los conocimientos en un mismo caso clínico, siguiendo el mismo patrón que utilizamos en el CIMA previo. Con ello, intentamos que el alumno genere conexiones entre toda la información recibida y trabajada. El alumno trabajará en grupos pequeños.

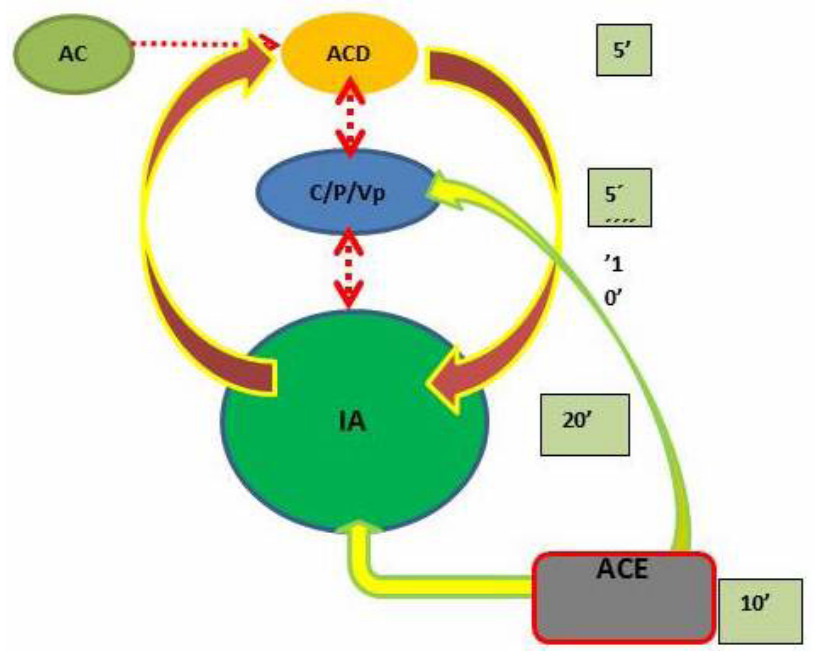

AC: Actividades de contraste previas a la asistencia al aula

ACD: Actividades de contrastedocentes (Indicaciones, explicaciones, etc) C/P/Vp: Caso/problema/video problema

LA: Ideas de los alumnos (trabajos en grupo pequeño (TGP), exposicióny debate entre grupos $(E+D)$, etc)

ACE: Actividades de contraste basadas en experimentos

Fig. 4 Modelo metodológico posible

El profesor plantea las preguntas claves al inicio de la actividad y explica la metodología a seguir para el trabajo autónomo del alumno mediante casos. A partir de 
ahí, juega un papel de moderador y guía de los equipos de trabajo, pasando el alumno a ser el protagonista de su aprendizaje. Posteriormente revisará el trabajo del alumno generando un feedback en el aprendizaje.

\section{Modificaciones introducidas sobre el CIMA previo}

Para llevar a cabo este modelo metodológico, hemos tenido que reestructurar los horarios del curso para disponer de más tiempo para realizar las actividades propuestas; hemos cambiado el orden de las actividades y modificado alguna de ellas, intentando que el alumno sea más autónomo en su aprendizaje. También, aunque en el curso anterior la asistencia a clase fue muy buena, este año se ha tenido en cuenta la asistencia, siendo necesario cubrir el $80 \%$ de las mismas (ya que en el aula se desarrollan muchas de las actividades de la asignatura), para ser consideradas en la nota final de la asignatura.

Los alumnos trabajaron en equipo durante todo el CIMA, equipos que son fijos desde el inicio del curso. Cada equipo está constituido por 5 alumnos.

Hemos intentado mejorar el control del tiempo, ya que fue quizás una de las mayores dificultades encontradas en el CIMA previo.

El alumno, se convierte en el protagonista de este CIMA. A pesar de llevarse a cabo en clases teóricas, en este CIMA no se imparte docencia magistral entendida como tal. El alumno es quien investiga, mediante el análisis de los casos clínicos, la materia que engloba los temas incluidos en este CIMA ampliado. El objetivo es aprender a reconocer estas patologías, diferenciarlas, y así poder plantear los tratamientos más adecuados de su especialidad.

Jornadas de Formación e Innovación Docente del Profesorado I № 2 (2019) Esta obra se distribuye con la licencia Creative Commons Reconocimiento-NoComercial-SinObraDerivada 
Tabla 1. Secuencia de actividades del nuevo CIMA

\begin{tabular}{|c|c|c|}
\hline $\begin{array}{l}\text { No DE } \\
\text { ACTIVIDAD }\end{array}$ & DESCRIPCIÓN DE LA ACTIVIDAD DIA 1 & TIEMPO \\
\hline$A C$ & $\begin{array}{l}\text { Actividades de contraste: Previo al inicio del } \\
\text { CIMA, se suben a plataforma los } 2 \text { temas a tratar, } \\
\text { para que el alumno repase la primera parte de } \\
\text { estos temas (recuerdo anatomofisiológico del } \\
\text { sistema vascular). }\end{array}$ & SIN TIEMPO \\
\hline \multirow[t]{2}{*}{$A C D$} & $\begin{array}{l}\text { El profesor reparte un cuestionario con } \\
1 \text { pregunta, "Cuál de estas pacientes es } \\
\text { susceptible de terapia manipulativa?. Explique } \\
\text { por qué”, referida a una imagen de una } \\
\text { diapositiva que se proyecta, a la que el alumno } \\
\text { contestará con una hipótesis personal, sin } \\
\text { consultar ninguna fuente. Lanzo las preguntas } \\
\text { que planteé en el mapa de contenido. La recoge } \\
\text { el profesor. }\end{array}$ & $3^{\prime}$ \\
\hline & $\begin{array}{l}\text { Distribuir en grupos de } 5 \text { alumnos y explicarles } \\
\text { la dinámica de trabajo. Numerar el grupo. } \\
\text { Repartir los casos clínicos basados en } \\
\text { diferentes tipos de edema. Entregar hoja de } \\
\text { reparto de roles, organización y seguimiento de } \\
\text { la actividad del grupo. }\end{array}$ & $7^{\prime}$ \\
\hline IA (TGP) & $\begin{array}{l}\text { Los alumnos trabajan en grupos reducidos ( } 5 \\
\text { alumnos) } \\
\text { Durante el resto del tiempo }\end{array}$ & $45^{\prime}$ \\
\hline Medios: & $\begin{array}{l}\text { Ordenador, proyector, papel, plataforma virtual, } \\
\text { papel y bolígrafo }\end{array}$ & \\
\hline $\begin{array}{l}\text { No }- \text { DE } \\
\text { ACTIVIDAD }\end{array}$ & DESCRIPCIÓN DE LA ACTIVIDAD DIA 2 & TIEMPO \\
\hline$A C D$ & $\begin{array}{l}\text { El profesor explica la dinámica de trabajo en el } \\
\text { aula. Exposición de casos }\end{array}$ & $1^{\prime}$ \\
\hline $\mathrm{Al}(\mathrm{E})$ & $\begin{array}{l}\text { Los alumnos salen por orden a exponer. (cada } \\
\text { equipo dispone de } 5 \text { minutos). El resto de } \\
\text { compañeros evalúan al equipo que expone. }\end{array}$ & $40^{\prime}$ \\
\hline
\end{tabular}

Jornadas de Formación e Innovación Docente del Profesorado | № 2 (2019) Esta obra se distribuye con la licencia Creative Commons Reconocimiento-NoComercial-SinObraDerivada Internacional (CC BY-NC-ND 4.0.) 


\begin{tabular}{|c|c|c|}
\hline $\mathrm{Al}(\mathrm{D})$ & $\begin{array}{l}\text { Se genera el debate entre todos los grupos } \\
\text { para hacer el diagnóstico diferencial de estas } \\
\text { patologías. El profesor detecta posibles errores } \\
\text { de concepto. Puntualizaciones y resumen del } \\
\text { profesor. }\end{array}$ & $15^{\prime}$ \\
\hline Medios: & Proyector, ordenador, pizarra, tiza & \\
\hline $\begin{array}{l}\text { No- DE } \\
\text { ACTIVIDAD }\end{array}$ & DESCRIPCIÓN DE LA ACTIVIDAD DIA 3 & TIEMPO \\
\hline$A C D$ & $\begin{array}{l}\text { El profesor explica la dinámica de trabajo en el } \\
\text { aula. }\end{array}$ & $1^{\prime}$ \\
\hline $\mathrm{Al}(\mathrm{D})$ & $\begin{array}{l}\text { Se revisan los casos, mediante imágenes que } \\
\text { trae el profesor. Debate y puntualizaciones. } \\
\text { Diagnóstico diferencial de estas patologías. }\end{array}$ & $45^{\prime}$ \\
\hline ACD & $\begin{array}{l}\text { Se vuelve a entregar la pregunta inicial para } \\
\text { evaluación de aprendizaje por el alumno y las } \\
\text { preguntas estructurantes. Las recoge el profesor }\end{array}$ & $7^{\prime}$ \\
\hline Medios: & Proyector, ordenador, pizarra, tiza & \\
\hline
\end{tabular}

\section{Diario de las Sesiones del nuevo CIMA}

En relación a la secuencia de sesiones realizadas en el curso anterior, y sobre el proyecto inicial que se ideó para este curso, se han realizado algunas modificaciones, intentando que el alumno sea más autónomo en su aprendizaje.

Dos días previos al inicio de la actividad, se sube a plataforma una nota informativa a los alumnos con el contenido del taller y el material que van a requerir parar en clase, además de los temas sobre los que vamos a trabajar.

Las clases se imparten en 2 grupos diferentes en las mismas fechas. El grupo B tiene clase de 11 a 12 horas, y el grupo $A$, de 13 a 14 horas. Yo imparto la docencia en ambos cursos. Dado que el contenido y la metodología es la misma para ambos grupos, se comentarán en conjunto. Si existían diferencias dignas de mención entre ambos grupos, se exponían aparte. 
En los días 28 al 31 de octubre, y el 4,5 y 14 de noviembre, se ha impartido el primer CIMA (el mismo del curso anterior), y los días 18 a 20 de noviembre la ampliación del CIMA. Al contrario que el año pasado, en este curso ya los alumnos no han preguntado si no se van a impartir clases estrictamente teóricas (probablemente ya se ha corrido la voz en la Facultad). Desde el inicio del cuatrimestre, han trabajado con diferentes métodos de innovación y lo han recibido muy bien. Desde el primer día de clase, se han distribuido en equipos de trabajo, que se mantienen a lo largo de toda la asignatura. La constitución de los equipos la han decidido los propios alumnos de forma consensuada, y posteriormente comunicado a los profesores.

La media de los alumnos que han asistido a clase, (hemos de tener en cuenta que la asistencia es obligatoria en el $80 \%$ de las clases con sus actividades correspondientes para poder incluirse en el grupo de evaluación continua), es de 29-31 alumnos en el grupo B y de 32-33 alumnos en el grupo A. En ningún caso, el número de alumnos fue inferior.

En ambas aulas, se han seguido las secuencias de actividades, tal como se especifican en la tabla 1. El primer día de este CIMA, se pasa un cuestionario con las preguntas estructurantes que se especifican en el mapa de contenidos, que servirán de control para la evaluación del proceso de aprendizaje. En función del tema a tratar, días previos a las clases, se les sube material que deben leer al menos, antes de llegar al aula.

Al llegar a clase se les daba las pautas de trabajo del día y comenzaban a trabajar en equipo. Se trabajaba a partir de casos clínicos, que o bien se proyectan en un vídeo, o bien se les suministraba mediante un simulacro, como si de una historia clínica se tratase.

A su vez, se les pide que asignen los roles de trabajo dentro del equipo. Han de rellenar una hoja, sobre el desarrollo del trabajo en equipo, el reparto de dichos roles 
(líder, secretario, gestor de tiempo), la organización que han seguido para hacer la búsqueda de la información y las fuentes consultadas para obtener dicha información.

En algunos temas, se les añade un cuestionario guía para orientar la dinámica de trabajo. A medida que vamos avanzando, se le da libertad al alumno para que esa guía la decida él mismo.

En el aula, mientras ellos trabajan, yo iba revisando los equipos para hacer mi propia valoración del trabajo y del grupo y aclarando las dudas que se presentaban; Por otro lado, ayudaba a los grupos que tenían dificultades para avanzar.

Además, posteriormente a la realización del trabajo, tenían que exponerlo. Con ello trabajábamos algunas aptitudes que debe tener este futuro profesional. Se les da un tiempo limitado para la exposición y la libertad de utilizar los medios que quieran para presentarla. El objetivo de esta presentación es conocer cómo han estructurado y organizado el trabajo dentro del equipo, como han buscado la información, a que conclusiones han llegado, que objetivos de tratamiento y qué reto se han planteado para su paciente. Es decir, trasladar al aula, algo de la actividad profesional que tendrán que realizar cuando salgan al mercado laboral.

Estas exposiciones, además del profesor, eran evaluadas por sus propios compañeros. Los ítems a valorar fueron: contenido, exposición, métodos utilizados y capacidad de transmitir.

Con la valoración, fomentamos por un lado, la intención del alumno de hacerlo lo mejor posible por ser evaluado, e incluso a veces, esta evaluación constituye un discreto elemento competitivo, muy eficaz en determinados alumnos, y por otro, el saber diferenciar los puntos 
clave que hay que tener en cuenta a la hora de transmitir información.

Además, cada equipo debía hacer al menos una pregunta al grupo que estaba realizando la exposición, ello les obligó a estar pendiente de los que decían sus compañeros, mejorando la atención y el silencio en clase a la hora de las presentaciones. Trabajamos con ello el respeto a los demás en el aula.

En las exposiciones, un factor a tener en cuenta es que los equipos se ajustasen al tiempo previsto lo más posible. Con ello, trabajamos la gestión del tiempo, uno de los problemas más dificiles a la hora de implantar innovaciones docentes en el aula.

Tras la exposición se generaban debates entre los propios alumnos. En general, han sido muy participativos y curiosos. Yo iba anotando los errores que detectaba, para aclararlos posteriormente.

En algunos temas, como conclusión final, los alumnos han trabajado en una cartulina, sus conclusiones sobre lo aprendido y los objetivos que han establecido para esas enfermedades.

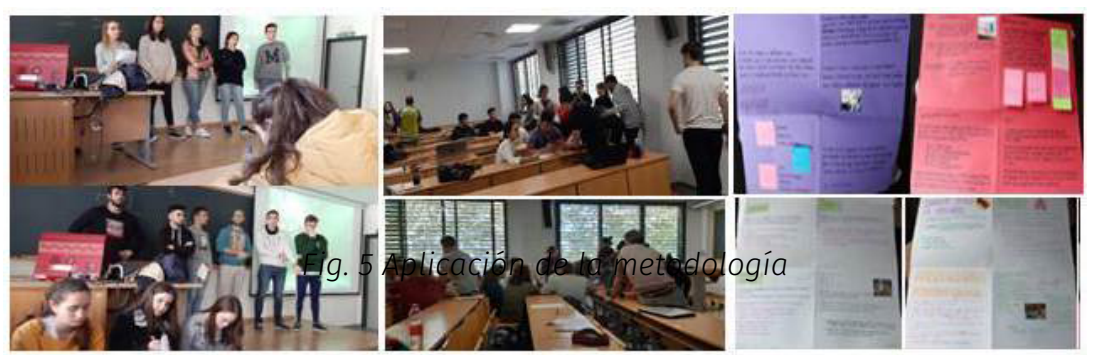

Fig. 5 Aplicación de la metodología

En otros temas, fundamentalmente los correspondientes a la ampliación del CIMA, la última parte del tiempo se ha dedicado a las conclusiones de los temas y a la 
aclaración de dudas, mediante sesión de imágenes que aludian a las patologías estudiadas. En esta actividad, de nuevo los alumnos fueron muy participativos, si bien, al no haber leído el material colgado en la plataforma previamente, la clase se ralentiza mucho porque hay que aclarar muchas dudas.

La evaluación, mediante la encuesta de las preguntas estructurantes para conocer cómo ha evolucionado el aprendizaje de los alumnos, prevista para el final de la última clase, se ha tenido que posponer por falta de tiempo hasta la siguiente, que será dentro de una semana. Esto hace imposible poder incorporar en este artículo las escaleras de aprendizaje.

Impresión del profesor sobre la experiencia: "Coincidiendo con los resultados del CIMA anterior, observo que los alumnos trabajan muy bien en equipo. Valoran mucho esta forma de trabajar. Durante todo el tiempo han estado buscando información. Ninguno estaba distraído en otras cosas, y eso qué, en alguna ocasión, la clase ha precedido a un examen esa misma tarde y a pesar de ello, la asistencia ha sido la misma. La experiencia ha sido muy positiva en ambos grupos.

Los alumnos trabajan bien también fuera del aula, ya sea reuniéndose fisicamente con su grupo, o a través de internet (foro, correo electrónico, etc). Suelen hacerlo para preparar trabajos o exposiciones.

Curiosamente, en el caso de las exposiciones, la experiencia en las 2 aulas ha sido diferente. El comportamiento a la hora de completar el trabajo y exponer ha sido muy distinto.

Un curso, salvo alguna excepción, ha expuesto en general con poca preparación del caso, con escasos medios 
(generalmente leyendo lo escrito) y con poca organización en el reparto de tareas.

En cambio, el otro curso, en esta ocasión, merece mención aparte, ya que, la mayoría han hecho una exposición muy elaborada, con una muy buena preparación y un material audiovisual muy adecuado, estando en las mismas circunstancias que el otro aula. Es llamativo, como los comportamientos en los distintos equipos de una misma aula son semejantes. Se potencian entre sí, tanto para lo bueno como para lo malo.

El método utilizado me ha permitido detectar con cierta facilidad los alumnos que destacan en cada grupo, tanto académicamente, como en liderazgo. Se ve claramente si el peso del trabajo en el grupo está repartido, o recae en alguno de los componentes exclusivamente.

Me he sentido bien con la experiencia, y me ha sorprendido gratamente cómo muchos de estos alumnos tienen una gran capacidad de trabajo.

Este cambio de metodología que considero muy eficaz, a veces no da los resultados esperados en el tiempo previsto, ya el compromiso del alumno, de trabajar fuera del aula parte del contenido para agilizar la clase, no se cumple en muchos casos. Sobre todo al principio, cuando la asignatura es de primer cuatrimestre y es la primera vez que los alumnos entran en contacto con otra forma de aprender. Conseguir este compromiso es, quizás, lo más complicado. Si no se alcanza, precisamos de más tiempo para que el alumno vaya entendiendo esta nueva forma de trabajar. No obstante, los alumnos refieren que la innovación docente hace las clases más amenas, divertidas y que el trabajo en equipo favorece el conocimiento y la integración de todos los alumnos en el aula, además de facilitarles el trabajo. La consideran muy positiva.

Jornadas de Formación e Innovación Docente del Profesorado | № 2 (2019) Esta obra se distribuye con la licencia Creative Commons Reconocimiento-NoComercial-SinObraDerivada 
El cambio realizado respecto a la participación de todos los componentes del grupo en la exposición, y que el resto de los alumnos evalúe a sus compañeros, ha conseguido involucrar aún más a la clase.

Sigo siendo una ferviente seguidora de innovar en el aula, porque el comportamiento de los alumnos es muy bueno. Se saca lo mejor de ellos y la relación interpersonal entre alumnos y profesor favorece el acercamiento y el aprendizaje.

El alumno, al trabajar de esta manera, se relaja y participa más y además el pertenecer a un grupo le protege y a la vez le empodera, al sentirse que forma parte útil de él. Además, ayuda a la integración del alumnado, incluso entre alumnos con ciertas dificultades sociales, pues se sienten considerados".

\section{Seguimiento de la Evolución:}

La evaluación del proceso de aprendizaje es primordial. Es la única manera de saber que está ocurriendo realmente con los alumnos y nos va a permitir mejorar la docencia.

Para ver la evolución en el aprendizaje de los alumnos, se realizó, cómo puede observarse en la secuencia de actividades, un cuestionario inicial para conocer los conocimientos de partida del alumno y este mismo cuestionario se pretendía pasar al finalizar este CIMA, para conocer la evolución del aprendizaje tras el ciclo.

El cuestionario inicial, nos permitió adecuar los contenidos a las necesidades reales de nuestros alumnos y el cuestionario final nos ayudará a comprobar cómo de eficaz ha sido el CIMA en el proceso de aprendizaje. 
Además de ello, evaluamos cómo han desarrollado y planteado los casos clínicos que se han trabajado en el aula, creando un feed-back con el alumno en la revisión y corrección del material preparado.

Pretendíamos utilizar las escaleras de aprendizaje. Estas escaleras, bien elaboradas, pueden darte mucha información tanto de forma grupal como individual de los alumnos.

Para llevar a cabo la evaluación de estos cuestionarios se aplicará una metodología consistente en agrupar las respuestas, ordenarlas por su nivel de complejidad, analizarlas buscando obstáculos y establecer conclusiones.

Desafortunadamente, los motivos explicados en el apartado anterior, no me han permitido poder completar por ahora, el seguimiento de la evolución del aprendizaje de mis alumnos. Se llevará a cabo sin duda, porque soy consciente de la importancia de este trabajo, pero los plazos de presentación estipulados desde la REFID no ha hecho posible que pueda completar esta tarea.

No obstante, cuando trabajas tantas horas con tus alumnos, y tienes la suerte de tener grupos no demasiado numerosos, te permite tomar con bastante certeza, la temperatura del aula. Vas viendo como los alumnos van evolucionando, no solo en conocimientos, sino en aptitudes y actitudes qué, en esta área de conocimiento, son tremendamente importantes.

\section{Evaluación del Diseño}

Considero que es fundamental la autoevaluación del profesor sobre su propio diseño didáctico y su actuación en el aula. Es un proceso de reflexión que te enriquece de manera importante y potencia el cambio. Así mismo, 
te permite ajustar las actividades diseñadas, teniendo en cuenta posibles fallos y errores identificados en el transcurso del CIMA.

Para llevar a cabo esta evaluación del diseño programado, me ha sido de gran utilidad mi mayor descubrimiento en el CIMA previo, el diario del profesor. Es una herramienta espectacular para autoevaluar la actividad realizada y validar los métodos utilizados. Así mismo, te permite de manera objetiva, ver errores cometidos y posibles mejoras. Si nos acostumbramos a registrar toda nuestra actividad en el aula, esto nos facilita tremendamente el conocer donde radican nuestros fallos, ver que está funcionando y es útil y reprogramar nuestra docencia haciéndola más acorde a las necesidades de nuestros alumnos.

Aunque también considero muy útil el hacer un portafolio que le permita al alumno tener de forma conjunta todas las actividades que ha realizado, y de camino le sirva de esquema para repasar lo aprendido, este curso no ha sido posible ponerlo en marcha. Es un objetivo futuro.

Otro pilar importante en la evaluación del diseño, es la opinión de los receptores, es decir, de los alumnos. Para ello, suelo pedir una encuesta de opinión al final del curso, para que el alumno exprese su opinión sobre el desarrollo de los ciclos de mejora, sus ventajas, inconvenientes y posibles sugerencias. Lamentablemente, tampoco puedo presentar aquí los resultados. A toro pasado, comentar que la evaluación del alumnado que experimentó el CIMA en el curso anterior, fue muy positiva, valorando mucho estas mejoras docentes. 


\section{Bibliografia}

Bain, K. (2007). Lo que hacen los mejores profesores universitarios. Valencia. Universidad de Valencia

Escribano, A y Del Valle, A. (2008). El aprendizaje basado en problemas. Una propuesta metodológica en Educación superior. Madrid. Narcea.

Finkel, D. (2008). Dar clase con la boca cerrada. Valencia. Universidad de Valencia

Porlán, R. (Coord.) (2017). Enseñanza Universitaria. Cómo mejorarla. Madrid. Morata

Porlán, R. y Martín, J. (1991): El diario del Profesor. Un recurso para la investigación en el aula. Sevilla. Díada.

Jornadas de Formación e Innovación Docente del Profesorado | № 2 (2019) Esta obra se distribuye con la licencia Creative Commons 